[Radiocirbon, V'ol. 20, No. 3, I978, P. 502-512]

\title{
UNIVERSITY OF MIAMI RADIOCARBON DATES XIII
}

\author{
P M CALVERT, D S INTRONE, and J J STIPP
}

Department of Geology, University of Miami

Coral Gables, Florida 33124

The following radiocarbon clates are a partial list of samples measured since Sept 1977. The chemical and counting procedures are the same as indicated previously (R, 1978, v 20, p 134-138).

Dates are conventional calculations based on the 5568-year I Libby half-life, uncorrected for isotopic fractionation in nature. $\delta^{13} \mathrm{C}$ is reported, however, for all samples analyzed for stable $\mathrm{G}$ isotope ratios. Errors are reported as one-standard deviation which include only the uncertainties of measurement of the modern NBS standard, background, and sample.

\section{ACKNOWLEDGMENTS}

Kim Rudolph instituted and carried out procedures for calcitearagonite measurements in carbonate samples.

\section{SAMIPIE DESC:RIPTIONS \\ I. ARCHAEOLOGIC SAMPLFS \\ United States}

\section{Western Columbia County series}

Charred wood from posts of Weeden Island ceremonial structure which date time of construction. Structure was razed and covered with Mound B cap after interment of an individual. Coll and subm Oct 1977 by J Milanich, Florida State Mus, Gainesville, Floricla.

\section{UM-1234. Charred Post II $1580 \pm 80$ \\ UM-1235. Charred Post I

$$
1490 \pm 80
$$ \\ UM-1260. Pinellas Co, Florida

$$
\begin{array}{r}
\mathbf{4 0 5 0} \pm \mathbf{8 0} \\
\delta^{I s} C=-26.01 \%
\end{array}
$$

Charcoal from $50 \mathrm{~cm}$ deep in shell midden found under overturned whelk shell, used to date habitation of site on Boca Ciega Bay $\left(27^{\circ} 49^{\prime} \mathrm{N}\right.$, $\left.82^{\circ} 50^{\prime} \mathrm{W}\right)$. Coll and subm 1977 by Ci Braley, Florida State Univ, Tallahassee.

\section{Newcomb Hearth series}

Charcoal from 4 hearth sites in Newcomb, New Nexico $\left(36^{\circ} 16^{\prime} 39^{\prime \prime}\right.$ $\mathrm{N}, 108^{\circ} 42^{\prime} 22^{\prime \prime} \mathrm{W}$ ) were studied to correlate and define prehistoric agricultural activities in area. Coll 1977 and subm 1978 by S Koczan, Mus New Mexico, Santa Fe. 
UM-1292. Grid 81

$$
\begin{array}{r}
1510 \pm 80 \\
\delta^{13} C=-12.64 \% o
\end{array}
$$

Charcoal from circular hearth, $65 \mathrm{~cm}$ diam, containing 4 round stones with no diagnostic artifacts or assoc architecture.

\section{UM-1293. Feature 11}

$$
\begin{array}{r}
1520 \pm 140 \\
\delta^{13} C=-15.80 \%
\end{array}
$$

Charcoal from hearth assoc with small habitation structure and ceramic assemblage of $800 \mathrm{BP}$.

\section{UM-1294. Feature 14}

$$
\begin{array}{r}
1090 \pm 90 \\
\delta^{13} \mathrm{C}=-11.82 \%
\end{array}
$$

Charcoal from circular hearth, $30 \mathrm{~cm}$ diam, with no assoc architecture.

UM-1295. Feature 10

$$
\begin{array}{r}
1240 \pm 60 \\
\delta^{13} \mathrm{C}=-12.39 \% \mathrm{C}
\end{array}
$$

Charcoal from rectangular hearth definable by 4 upright sandstone slabs; no assoc architecture.

\section{Hardee Co Firepit series}

Charcoal from 2 aboriginal firepits in Baird Quad in NW Hardee Co, Florida $\left(27^{\circ} 38^{\prime} 45^{\prime \prime} \mathrm{N}, 81^{\circ} 57^{\prime} 0^{\prime \prime} \mathrm{W}\right)$. Coll June 1977 by B Wharton, Univ South Florida and subm Sept 1977 by R Williams, Univ South Florida, Tampa. Comment (RW): useful in interpreting portion of Central Gulf Coast and Manatee archaeol regions.

UM-1198. 8Hr11/B59/2

$$
\mathbf{2 0 7 0} \pm \mathbf{7 0}
$$

Charcoal from top layer of firepit found $20 \mathrm{~cm}$ below ground surface. Assoc potsherds of ceramic vessel.

UM-1199. 8Hr11/B59/3

From same zone as UM-1198.

$$
\begin{array}{r}
\mathbf{1 7 3 0} \pm \mathbf{8 0} \\
\delta^{13} C=-25 \% \\
\mathbf{1 7 1 0} \pm \mathbf{1 3 0} \\
\delta^{13} C=-26.58 \%
\end{array}
$$

UM-1200. 8Hr11/B59/1 -1199 .

Charcoal from $28 \mathrm{~cm}$ below surface in same firepit as UM-1198 and

\section{UM-1201. 8Hr11/B1/1.}

$$
\begin{array}{r}
1270 \pm 70 \\
\delta^{13} C=-26.36 \%
\end{array}
$$

Charred wood from $15 \mathrm{~cm}$ below surface in firepit containing chipped stone and pottery fragments.

UM-1202. 8Hr11/B1/2

From same zone as UM-1201.

\section{Makran Coast series}

$$
\text { II. GEOLOGIG SAMPIES }
$$

$$
\text { A. Iran }
$$

$$
\begin{array}{r}
1170 \pm 90 \\
\delta^{13} C=-26.50 \%
\end{array}
$$

Studies using shell samples from sites on Makran coast indicate amount Quaternary deformation varies greatly geographically. Degree 
of movement as well as rates of movement are being studied from age/ height values (Vita-Finzi, 1975; 1978). Coll and subm 1976 by G VitaFinzi, Univ College, London, England.

UM-1256. PP(a)

$20,560 \pm 250$

$\delta^{13} \mathrm{C}=-1.45 \%$

Anadara uropigmelana from fossil beach $\left(25^{\circ} 09^{\prime} \mathrm{N}, 61^{\circ} 13^{\prime} \mathrm{E}\right) \mathrm{ca}$ $+50 \mathrm{~m}$ for comparison to UM-1145 at similar height.

\section{UM-1145. Beris 1A}

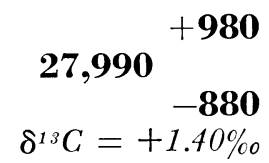

Anadara uropigmelana from coquina layer, $1 \mathrm{~m}$ thick $\left(25^{\circ} 11^{\prime} \mathrm{N}\right.$, $\left.61^{\circ} 11^{\prime} \mathrm{E}\right) \mathrm{ca}+50 \mathrm{~m}$.

UM-1160. Beris 1G

$$
\begin{gathered}
\mathbf{2 8 , 5 6 0} \\
\delta^{1 s} C=-0.59 \% \\
-\mathbf{5 3 0 0}
\end{gathered}
$$

Gastropods from coquina layer, $1 \mathrm{~m}$ thick $\left(25^{\circ} 11^{\prime} \mathrm{N}, 61^{\circ} 11^{\prime} \mathrm{E}\right) \mathrm{ca}$ $55 \mathrm{~m}$ above high water exposed by narrow gullies.

\section{UM-1148. CB1 L}

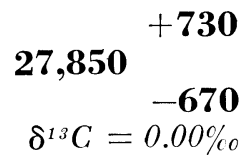

Oyster shell from beach deposit $\left(25^{\circ} 16^{\prime} \mathrm{N}, 60^{\circ} 37^{\prime} \mathrm{E}\right) 10 \mathrm{~cm}$ thick and $16.65 \mathrm{~m}$ above high water.

UM-1149. K76C (G)

$$
\begin{array}{r}
\mathbf{5 7 2 0} \pm \mathbf{8 0} \\
\delta^{18} C=+1.13 \%
\end{array}
$$

Gastropod fragments from fossil beach $\left(25^{\circ} 19^{\prime} \mathrm{N}, 60^{\circ} 23^{\prime} \mathrm{E}\right)$ in $25 \mathrm{~cm}$ thick layer on exposed cliff face $17.75 \mathrm{~m}$ above high water.

UM-1146. K76C (B)

$$
\begin{gathered}
\mathbf{2 4 , 5 9 0} \\
\delta^{1.8} C=-0.59 \% \\
-\mathbf{8 7 0}
\end{gathered}
$$

Balanus from fossil beach $\left(25^{\circ} 19^{\prime} \mathrm{N}, 60^{\circ} 23^{\prime} \mathrm{E}\right)$ in pebble layer, $25 \mathrm{~cm}$ thick, under $3.6 \mathrm{~m}$ cross-bedded sand $17.75 \mathrm{~m}$ above high water.

UM-1147. K76 (O)

Oyster shell from fossil beach $\left(25^{\circ} 19^{\prime} \mathrm{N}, 60^{\circ} 23^{\prime} \mathrm{E}\right)$ in pebble layer, $25 \mathrm{~cm}$ thick, in cliff face $17.75 \mathrm{~m}$ above high water.

UM-1150. E Jask (1)

$6620 \pm 130$

Anadara uropigmelana from fossil beach $\left(25^{\circ} 39^{\prime} \mathrm{N}, 57^{\circ} 46^{\prime} \mathrm{E}\right) \mathrm{lm}$ above high water that grades upward into alluvial terrace. 
UM-1151. E Jask (2)

Oliva sp from same site as UM-1150.

UM-1159. Jask 1G
$4870 \pm 100$
$\delta^{13} C=+0.09 \%$

$19,170 \pm 380$

$\delta^{13} \mathrm{C}=+0.12 \%$

Gastropods from coquina and sand, $10 \mathrm{~cm}$ thick, surface layer in fossil beach $\left(25^{\circ} 39^{\prime} \mathrm{N}, 57^{\circ} 48^{\prime}\right.$ E) $6 \mathrm{~m}$ above high water.

UM-1255. Tujak III (x)

$$
6160 \pm 160
$$

$\delta^{13} C=-0.48 \%$

Asaphis deflorata from pebbly marl layer, $1 \mathrm{~m}$ thick $\left(26^{\circ} 01^{\prime} \mathrm{N}, 57^{\circ}\right.$ $\left.14^{\prime} \mathrm{E}\right)+28.6 \mathrm{~m}$.

UM-1268. Tujak II(b)

$3310 \pm 90$

$\delta^{13} \mathrm{C}=+0.79 \%$

Anadara uropigmelana in pebbly marl $\left(26^{\circ} 01^{\prime} \mathrm{N}, 57^{\circ} 14^{\prime} \mathrm{E}\right)+11.8 \mathrm{~m}$.

UM-1269. Tujak III(b)

$6110 \pm 100$

$\delta^{13} \mathrm{C}=+0.46 \%$

Anadara uropigmelana from pebbly marl layer $\left(26^{\circ} 01^{\prime} \mathrm{N}, 57^{\circ} 14^{\prime} \mathrm{E}\right)$ $+28.6 \mathrm{~m}$.

\section{Laguna Inferior series}

\section{B. Mexico}

Marine shells from barrier dune of Laguna Inferior $\left(16^{\circ} 15^{\prime} 30^{\prime \prime} \mathrm{N}\right.$, $94^{\circ} 36^{\prime} 40^{\prime \prime} \mathrm{W}$ ) coll Sept 1977 and subm Dec 1977 by A Carranza-Edwards, Univ Naci Autonoma Mexico, Mexico 20, DF, to study past sea level at site $20 \mathrm{~m} \mathrm{E}$ of Laguna Inferior and $+7 \mathrm{~m}$. One sample was divided into 3 parts.

UM-1262. A

$$
\begin{array}{r}
1150 \pm \mathbf{8 0} \\
\delta^{13} C=-2.19 \%
\end{array}
$$

UM-1263. C

$1340 \pm 90$

UM-1264. D

$$
\delta^{13} \mathrm{C}=-1.82 \%
$$

\section{Laguna Mar Tileme series}

$1030 \pm 70$

Marine shells from barrier dune of Laguna Mar Tileme $\left(16^{\circ} 14^{\prime} 10^{\prime \prime}\right.$ N, 94 50' 50" W) coll Sept 1977 and subm Dec 1977 by A CarranzaEdwards to study past sea level. Sample taken S of Laguna Mar Tileme, at $+7 \mathrm{~m}$; divided into 3 parts.

UM-1265. B

$$
\begin{array}{r}
\mathbf{2 0 9 0} \pm \mathbf{1 0 0} \\
\delta^{13} C=-2.37 \% \text { o }
\end{array}
$$

UM-1266. $\quad$ E

$$
2100 \pm 90
$$

UM-1267. F

$$
\delta^{13} \mathrm{C}=-1.21 \%
$$

$\mathbf{2 3 8 0} \pm \mathbf{8 0}$

$\delta^{13} C=-0.50 \%$ 


$$
\text { C. Algerian Slope }
$$

Calcareous sediments from Algerian Slope for correlation of carbonate and temperature curves in Western Mediterranean Basin. Coll Sept 1976 and subm Oct 1977 by P Loubere, Oregon State Univ, Corvallis, Oregon

\section{UM-1 187. TR 173-7P}

$$
26,300 \pm 700
$$

From core $\left(36^{\circ} 56^{\prime} \mathrm{N}, 2^{\circ} 25^{\prime} \mathrm{E}\right) 484 \mathrm{~cm}$ long, $-1719 \mathrm{~m}$.

\section{UM-1212. TR 173-10P}

\section{8,450}

From core $\left(37^{\circ} 34.9^{\prime} \mathrm{N}, 00^{\circ} 09.4^{\prime} \mathrm{E}\right) 736 \mathrm{~cm} \mathrm{long},-1108 \mathrm{~m}$

UM-1213. TR 173-16P

$22,060 \pm 360$

From core $\left(36^{\circ} 10.1^{\prime} \mathrm{N}, 1^{\circ} 51.4^{\prime} \mathrm{W}\right) 710 \mathrm{~cm}$ long, $-1904 \mathrm{~m}$.

\section{Carrie Bow Cay series}

\section{Belize}

Four cores containing peat, marine and, terrigenous sediments coll on Belize shelf. Peat, predominantly Rhizophora, was dated to determine submergence curve for Belize shelf. Cores CB7 (16 $54^{\prime} 15^{\prime \prime} \mathrm{N}, 88^{\circ} 15^{\prime} 50^{\prime \prime}$

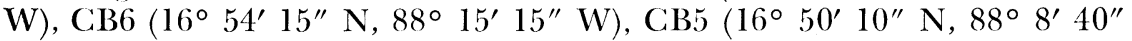
$\mathrm{W})$ and CB2 $\left(16^{\circ} 47^{\prime} 50^{\prime \prime} \mathrm{N}, 88^{\circ} 06^{\prime} 30^{\prime \prime} \mathrm{W}\right)$ are piston cores obtained with scuba. Coll 1977 by E Shinn; subm 1977 by D S Introne, Univ Miami, Miami, Florida. Depths given are below present day mean sealevel.

General Comment (DSI): acid washing only due to nature and size of samples.

UM-1248. CB7-B-A

$5.19 \mathrm{~m}$.

UM-1249. CB5-B-A

$13.97 \mathrm{~m}$

UM-1250. CB5-C-B

$14.86 \mathrm{~m}$.

UM-1251. CB6-A-B

$\mathrm{NaOH}$ pretreatment, $-7.33 \mathrm{~m}$.

UM-1252. CB6-A-A

$7.01 \mathrm{~m}$.

UM-1310. CB2-A

$9.61 \mathrm{~m}$

$$
\begin{array}{r}
2860 \pm 100 \\
\delta^{13} C=29.16 \% \\
\mathbf{7 6 4 0} \pm \mathbf{1 6 0} \\
\delta^{13} C=-27.90 \% \\
\mathbf{8 2 4 0} \pm \mathbf{1 4 0} \\
\delta^{13} C=-29.62 \% \\
\mathbf{8 8 5 0} \pm \mathbf{3 5 0} \\
\delta^{13} C=-27.52 \% \\
\mathbf{6 8 1 0} \pm \mathbf{8 0} \\
\delta^{13} C=-27.23 \% \\
\mathbf{6 4 3 0} \pm \mathbf{7 0} \\
\delta^{13} C=-26.11 \% 0
\end{array}
$$


UM-1311. CB2-B

$7520 \pm 70$

$10 \mathrm{~m}$.

$\delta^{13} C=-27.42 \%$ o

UM-1312. CB2-C

$7340 \pm 80$

$10.12 \mathrm{~m}$.

$\delta^{1.3} \mathrm{C}=-27.94 \%$

UM-1313. CB2-A

$5010 \pm 70$

Shell material incorporated within UM-1310. Comment (DSI): younger age implies shell material to be of burrowing variety.

UM-1314. CB2-C

$4250 \pm 60$

Shell material. Comment (DSI): younger age implies shell material to be of burrowing variety since it is assoc with UM-1312.

\section{Orange Cay series}

$$
\text { E. Bahamas }
$$

Peliod sand cored from Orange Cay $\left(24^{\circ} 56^{\prime} 45^{\prime \prime} \mathrm{N}, 79^{\circ} 8^{\prime} 03^{\prime \prime} \mathrm{W}\right.$ ) studied to determine if sand in marine shoal under aeolian dune is Holocene of late Pleistocene sea level high. Coll Aug 1977 and subm Sept 1977 by D Beach and R Ginsburg, Univ Miami, Fisher Island Sta, Miami Beach, Florida.

UM-1188. OJ-3-45

$14 \mathrm{~m}$ from core surface.

UM-1189. OJ-3-60

$18 \mathrm{~m}$ from core surface.

$$
34,830 \pm 680
$$

$$
38,650+1120
$$

\section{Joulters Cay Bryozoan series}

Several samples of bryozoan rock, lithothaminoid rock, and consolidlated ooid rock hand-picked from submerged reef in Joulters Cay, Bahamas $\left(25^{\circ} \mathrm{N}, 78^{\circ} \mathrm{W}\right)$. Samples coll to determine regional history, development, and diagenesis of bryozoan reefs. Coll by R J Cuffey, Penn State Univ; subm 1977 by M Yukon, Univ Miami, Florida.

UM-1214. OCR-D-4

$1740 \pm 110$

Consolidated ooid, $5 \mathrm{~m}$ depth.

UM-1215. NWN-A-1

$2590 \pm 100$

Bryozoan rock encrusted upon consolidated ooid, $5 \mathrm{~m}$ depth.

UM-1216. OCR-D-2

$2990 \pm 100$

Bryozoan encrusting trepostomes and cyclostomes infilled with ooids. Coll $4 \mathrm{~m}$ below high water.

UM-1217. OCR-A-1

$2120 \pm 130$

Lithothamnioid rock coll 3m below high water. 
UM-1218. OCR-D-1

$2060 \pm 80$

Bryozoan knob coll $4 \mathrm{~m}$ below high water.

Lithothamnioid rock coll from reef mass $3 \mathrm{~m}$ below surface.

UM-1219. CMM-A-1

$1730 \pm 90$

\section{F. United States}

\section{Cluett Key series}

Carbonate cores from Cluett Key $\left(25^{\circ} 01^{\prime} 54^{\prime \prime} \mathrm{N}, 80^{\circ} 51^{\prime} 42^{\prime \prime} \mathrm{W}\right)$ in Florida Bay studied to determine sedimentation rate on island and to ascertain that proto-dolomite in sediments is Holocene ( $\mathrm{R}, 1977, \mathrm{v} 19$, p 455-456). Coll June 1975 by R Halley, USGS, Miami Beach, R Steinen, Univ Connecticut, Storrs, and subm Mar 1978 by M Calvert.

UM-1296. C1-3-1-0

$1580 \pm 90$

Top of core to $2 \mathrm{~cm}$; no dolomite.

UM-1297. C1-3-29-0

$2620 \pm 100$

28 to $30 \mathrm{~cm}$; no dolomite.

UM-1298. C1-3-52-0

$2475 \pm 80$

51 to $53 \mathrm{~cm}$; no dolomite.

UM-1299. C1-3-89

$3100 \pm 100$

88 to $90 \mathrm{~cm}$; dolomite content not known.

UM-1300. C1-3-119

$2540 \pm 70$

118 to $120 \mathrm{~cm}$; dolomite content not known.

UM-1301. C1-3-160

$3420 \pm 100$

159 to $161 \mathrm{~cm}$; dolomite content not known.

UM-1302. C1-3-204-41

$3500 \pm 90$

203 to $205 \mathrm{~cm}$; sediment contains $41 \%$ dolomite.

UM-1303. C1-3-212

$3810 \pm 130$

211 to $213 \mathrm{~cm}$; dolomite content not known.

UM-1304. C1-3-214-32

$4020 \pm 90$

213 to $215 \mathrm{~cm}$; sediment contains $32 \%$ dolomite.

UM-1305. C1-3-260

$4310 \pm 90$

259 to $261 \mathrm{~cm}$; dolomite content not known.

UM-1306. C1-3-279-0

$6000 \pm 260$

278 to $280 \mathrm{~cm}$; no dolomite found in sediments which were laced with mangrove peat.

\section{Portsmouth Island series}

Peat and wood fragments for stratigraphic correlation of Back Bar- 
rier I., Diamond City and Core Creek sand formations, coll and subm 1977 by J Herbert, Duke Univ, Durham, North Carolina.

General Comment $(\mathrm{PC})$ : tops of cores are +1 to $+1.5 \mathrm{~m}$. Depth measurements are from top of core.

UM-1191. PB-6 (9.5m)

$\mathbf{7 7 8 0} \pm \mathbf{3 7 0}$

Wood chips in clay layer of Diamond City formation from site $\mathrm{N}$ of Isa Morris Camp ( $34^{\circ} 54^{\prime} 30^{\prime \prime} \mathrm{N}, 76^{\circ} 14^{\prime} 30^{\prime \prime} \mathrm{W}$ ).

UM-1192. PB-13 (19.8 to 21.3m)

$12,720 \pm 470$

Fibrous organics in clayey sand of Diamond City formation $\left(35^{\circ} 3^{\prime}\right.$ $\left.7^{\prime \prime} \mathrm{N}, 76^{\circ} 3^{\prime} 22^{\prime \prime} \mathrm{W}\right)$.

UM-1193. PB-4 (15.2m)

Peat in silty sand layer of Core Creek sand formation from site $\mathrm{N}$ of Drum Inlet ( $\left.34^{\circ} 53^{\prime} 45^{\prime \prime} \mathrm{N}, 76^{\circ} 15^{\prime} 45^{\prime \prime} \mathrm{W}\right)$.

UM-1194. PB-2 (6.5m)

$6500 \pm 160$

Wood in silty sand layer of Back Barrier I. formation from site $\mathrm{N}$ of Drum Inlet (34 $\left.52^{\prime} 15^{\prime \prime} \mathrm{N}, 76^{\circ} 17^{\prime} 30^{\prime \prime} \mathrm{W}\right)$.

UM-1195. PB-26 (1m)

$\mathbf{5 3 0} \pm \mathbf{8 0}$

Peat in fine sand layer from site near Merkle Hammock $\left(34^{\circ} 59^{\prime} 45^{\prime \prime}\right.$ N, $\left.76^{\circ} 8^{\prime} 15^{\prime \prime} \mathrm{W}\right)$.

UM-1196. PB-13 (14 to $15 \mathrm{~m}$ )

$7230 \pm 160$

Wood chips in silty, clayey sand layer of Back Barrier I. formation (35० $3^{\prime} 7^{\prime \prime} \mathrm{N}, 76^{\circ} 3^{\prime} 22^{\prime \prime} \mathrm{W}$ ).

UM-1197. PB-38 (17.7m) $\quad 25,220 \pm 480$

Wood fragments in peaty layer of Diamond City formation $\left(35^{\circ} 2^{\prime}\right.$ $\left.30^{\prime \prime} \mathrm{N}, 76^{\circ} 3^{\prime} 45^{\prime \prime} \mathrm{W}\right)$.

\section{UM-1282. PB-11 (18.3m)}

$$
30,470{ }^{+800}
$$$$
\delta^{13} \mathrm{C}=-29.88 \% \text { o }
$$

Wet peaty sand layer of Diamond City formation $\left(35^{\circ} 2^{\prime} 6^{\prime \prime} \mathrm{N}\right.$, $\left.76^{\circ} 4^{\prime} 50^{\prime \prime} \mathrm{W}\right)$.

UM-1283. PB-12 (12.5m)

$$
\begin{array}{r}
6560 \pm 260 \\
\delta^{13} C=-29.05 \% 0
\end{array}
$$

Wood chips in sandy layer of Back Barrier I. formation. Site $\left(35^{\circ} 3^{\prime}\right.$ $8^{\prime \prime} \mathrm{N}, 76^{\circ} 3^{\prime} 30^{\prime \prime} \mathrm{W}$ ) is $1.37 \mathrm{~km}$ from UM-1197 and $1.08 \mathrm{~km}$ from UM-1196 and -1192 . 


\section{UM-1284. PB-8 (19.8 to $21.3 \mathrm{~m}$ )}

Wood chips in sandy layer of Diamond City formation (34 $58^{\prime} 42^{\prime \prime}$ N, $76^{\circ} 09^{\prime} 45^{\prime \prime} \mathrm{W}$ ).

UM-1285. PB-21 (6.7 to $7.0 \mathrm{~m})$

$$
4830 \pm 170
$$

Wood in silty sand layer $1.13 \mathrm{~km}$ from UN-1194.

\section{Carolina Shoreline series}

Peat, shell, and wood, coll 1977 and subm 1978 by W J Cleary, Univ North Carolina, Wilmington, for sea-level curve and shoreline studies.

UM-1261. Caswell Pond 1

$$
\begin{array}{r}
\mathbf{3 8 7 0} \pm \mathbf{6 0} \\
\delta^{1.3} \mathrm{C}=-18.1+\%
\end{array}
$$

Freshwater peat, $4.9 \mathrm{~m}$ into core taken in pond at Caswell Reach (33 $54^{\prime} 35^{\prime \prime} \mathrm{N}, 78^{\circ} 04^{\prime} 35^{\prime \prime} \mathrm{W}$ ) in Pleistocene dune fields.

\section{UM-1274. Long Beach Motel}

$$
\begin{array}{r}
1510 \pm 90 \\
\delta^{13} C=-20.96 \%
\end{array}
$$

Freshwater peat outcropping on beach face at low tide at Long Beach $\left(33^{\circ} 53^{\prime} 30^{\prime \prime} \mathrm{N}, 78^{\circ} 07^{\prime} 00^{\prime \prime} \mathrm{W}\right)$.

UM-1275. Sample \#4 Wood

$$
\delta^{13} C=-27.10 \%
$$

Bay/Magnolia, id by $\mathrm{R}$ Thomas, removed from stump forest on

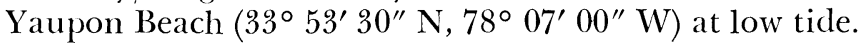

UM-1286.

Duplicate run of UM-1275.

UM-1276. Sample \#6 Wood

$$
\begin{array}{r}
\mathbf{2 5 3 0} \pm \mathbf{7 0} \\
\delta^{13} C=-26.99 \%
\end{array}
$$

$$
\begin{array}{r}
4060 \pm 90 \\
\delta^{13} C=-26.67 \%
\end{array}
$$

Southern Yellow Pine, id by $\mathrm{R}$ Thomas, removed from stump forest

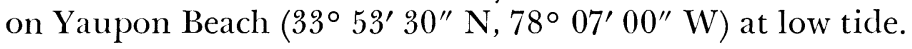

UM-1287.

Duplicate run of UM-1276.

UM-1288. Onslo Bch So Peat
$3820 \pm 80$ $\delta^{13} \mathrm{C}=-26.18 \%$

$$
\mathbf{3 8 0} \pm \mathbf{5 0}
$$

Salt-water peat (Spartina alteom), id by $\mathrm{P}$ Hosier, outcropping on Onslow Beach (34 $32^{\prime} 55^{\prime \prime}$ N, $77^{\circ} 19^{\prime} 28^{\prime \prime} \mathrm{W}$ ) at low tide.

\section{UM-1289. CBSB Line 3-1-160cm}

$$
\begin{array}{r}
\mathbf{2 7 2 0} \pm \mathbf{7 0} \\
\delta^{13} \mathrm{C}=-0.51 \%
\end{array}
$$

Oyster shells cored from below peat outcropping on beach face of Carolina Beach ( $\left.34^{\circ} 03^{\prime} \mathrm{N}, 77^{\circ} 53^{\prime} \mathrm{W}\right)$. 
UM-1290. CBSB 5-7 (145 to $150 \mathrm{~cm}$ )

$$
\begin{array}{r}
1930 \pm 70 \\
\delta^{13} C=-0.37 \%
\end{array}
$$

Bulk carbonate shells from below present marsh surface at Carolina Beach $\left(34^{\circ} 03^{\prime} \mathrm{N}, 77^{\circ} 53^{\prime} \mathrm{W}\right)$.

\section{Safety Valve series}

Several shell and Porites divasticata samples taken from hand pushpiston Core E of Soldier Key, Biscayne Bay, Florida $\left(25^{\circ} 35^{\prime} \mathrm{N}, 80^{\circ} 10^{\prime}\right.$ $\mathrm{W})$, to determine beginning of formation of safety valve tidal bar belt (UM-V, R, 1976, v 18, p 119-120). Coll and subm by J Sloan, RSMAS, Miami, Florida.

UM-1236. JLS-2:218 to $205 \mathrm{~cm}$

$$
\begin{array}{r}
\mathbf{2 0 5 0} \pm \mathbf{1 2 0} \\
\mathbf{2 3 8 0} \pm \mathbf{9 0} \\
\delta^{1 s} C=+2.02 \% \\
\mathbf{1 2 2 0} \pm \mathbf{8 0} \\
\delta^{13} C=+2.10 \% \\
\mathbf{1 9 0 0} \pm \mathbf{7 0} \\
\delta^{13} C=-0.67 \% \\
\mathbf{1 4 7 0} \pm \mathbf{1 0 0} \\
\mathbf{1 5 4 0} \pm \mathbf{9 0} \\
\delta^{13} C=+2.09 \%
\end{array}
$$

UM-1237. JLS-2:205 to $185 \mathrm{~cm}$

UM-1238. JLS-2:125 to $141 \mathrm{~cm}$

UM-1239. JLS-1:340 to $350 \mathrm{~cm}$

UM-1240. JLS-1:275 to $290 \mathrm{~cm}$

UM-1241. JLS-1:155 to $165 \mathrm{~cm}$

UM-1242. JLS-1:40 to $50 \mathrm{~cm}$

Porites divasticata.

$560 \pm 60$

\section{Card Sound series}

Red mangrove peat taken from hand push-piston core just $\mathrm{S}$ of Turkey Point canal ca $10 \mathrm{~km}$ W of Biscayne Bay $\left(25^{\circ} 20^{\prime} 35^{\prime \prime} \mathrm{N}, 80^{\circ} 20^{\prime}\right.$ W). Samples obtained for initial study to determine if peat accumulation destroys bedrock by physical and chemical processes. Coll and subm 1977 by J F Meeder, RSMAS, Miami, Florida.

General Comment (JMF): implications of study show that Red Mangrove peat overlying carbonate bedrock may not be used as paleo-sea-level indicator without rate of bedrock destruction; also, mangrove hammocks seem to cause depressions found beneath them rather than being caused by them.

UM-1226. JFM 3-1:220 to $222 \mathrm{~cm}$

Basal peat layer.

UM-1227. JFM 3-1A:210 to $217 \mathrm{~cm}$

UM-1228. JFM 3-2:187 to $195 \mathrm{~cm}$

$$
\begin{array}{r}
\mathbf{1 3 9 0} \pm \mathbf{8 0} \\
\delta^{13} C=-26.51 \% \\
\mathbf{2 6 2 0} \pm \mathbf{2 7 0} \\
\delta^{13} C=-26.07 \% \\
\mathbf{4 0 5 0} \pm \mathbf{7 0} \\
\delta^{13} C=-26.36 \%
\end{array}
$$


UM-1229. JFM 3-3:175 to $183 \mathrm{~cm}$

$$
\begin{array}{r}
\mathbf{3 1 7 0} \pm \mathbf{9 0} \\
\delta^{13} C=-26.67 \% \\
\mathbf{1 3 3 0} \pm \mathbf{7 0} \\
\delta^{13} C=-27.17 \% 0
\end{array}
$$

UM-1230. JFM 3-7:39 to $47 \mathrm{~cm}$

UM-1231. JFM 3-8:3 to $11 \mathrm{~cm}$

$4030 \pm 160$

\section{La Costa I series}

Sub-soil shell hash from La Costa I., Florida $\left(26^{\circ} 41^{\prime} 30^{\prime \prime} \mathrm{N}, 82^{\circ} 14^{\prime}\right.$ $\left.30^{\prime \prime} \mathrm{W}\right)$. Coll to determine relative sea-level changes in Florida and barrier island formation. Coll and subm by $\mathrm{T}$ Barron, Univ Miami, Florida.

UM-1220. SAM 1

$$
\begin{array}{r}
\mathbf{7 6 0} \pm \mathbf{8 0} \\
\delta^{13} C=-0.26 \% \\
\mathbf{1 1 4 0} \pm \mathbf{9 0} \\
\delta^{13} C=-0.52 \%
\end{array}
$$

UM-1221. SAM 2

UM-1222. SAM 5

UM-1223. SAM 4

$$
\begin{array}{r}
1880 \pm 90 \\
\delta^{13} \mathrm{C}=+0.03 \%
\end{array}
$$

UM-1224. SAM 3

$$
\mathbf{2 3 1 0} \pm \mathbf{8 0}
$$

$$
\delta^{13} \mathrm{C}=-0.02 \%
$$

UM-1225. SAM 6

$$
\begin{array}{r}
2230 \pm 90 \\
\delta^{13} C=-0.17 \% \\
1200 \pm 110 \\
\delta^{13} C=-0.33 \%
\end{array}
$$

REFERENCES

Piepgras, D, Calvert, M M, and Stipp, J J, 1978, University of Miami radiocarbon dates XI: Radiocarbon, v 20, p 134-138.

Piepgras, D and Stipp, J J, 1977, University of Miami radiocarbon dates VIII: Radiocarbon, v 19, p 118-126.

Stipp, J J, Eldridge, K L, and Hattner, J, 1976, University of Miami radiocarbon dates V: Radiocarbon, v 18, p 119-120.

Vita-Finzi, C, 1975, Quaternary deposits in the Iranian Makran: Geog Jour, v 141, p 415-420. Survey Iran Bull (in press). 